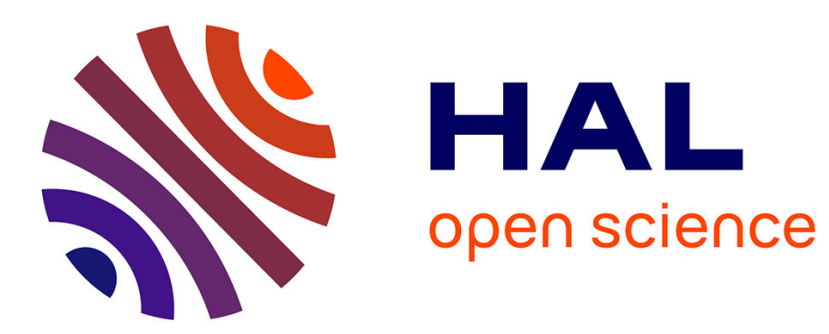

\title{
Multi-polarization quantum control of rotational motion through dipole coupling
}

\author{
Gabriel Turinici, Herschel Rabitz
}

\section{To cite this version:}

Gabriel Turinici, Herschel Rabitz. Multi-polarization quantum control of rotational motion through dipole coupling. Journal of Physics A: Mathematical and Theoretical, 2010, 43 (10), pp.105303. hal-00450794v3

\section{HAL Id: hal-00450794 \\ https://hal.science/hal-00450794v3}

Submitted on 9 Feb 2010

HAL is a multi-disciplinary open access archive for the deposit and dissemination of scientific research documents, whether they are published or not. The documents may come from teaching and research institutions in France or abroad, or from public or private research centers.
L'archive ouverte pluridisciplinaire HAL, est destinée au dépôt et à la diffusion de documents scientifiques de niveau recherche, publiés ou non, émanant des établissements d'enseignement et de recherche français ou étrangers, des laboratoires publics ou privés. 


\title{
Multi-polarization quantum control of rotational motion through dipole coupling
}

\author{
Gabriel Turinici \\ E-mail: Gabriel.Turinici@dauphine.fr \\ CEREMADE, Université Paris Dauphine, Place du Marechal De Lattre De Tassigny, \\ 75775 PARIS Cedex 16, France
}

\section{Herschel Rabitz}

E-mail: hrabitz@princeton.edu

Department of Chemistry, Princeton University, Princeton, NJ 08544-1009, USA

\begin{abstract}
In this work we analyze the quantum controllability of rotational motion under the influence of an external laser field coupled through a permanent dipole moment. The analysis takes into consideration up to three polarization fields, but we also discuss the consequences for working with fewer polarized fields.
\end{abstract}




\section{Introduction}

The manipulation of quantum phenomena is receiving increasing attention with laboratory demonstrations, including for control of rotational motion $[1,2,3,4,5,6]$. A fundamental issue underlying such experiments is a basic assessment of the possibility of attaining full control in any particular physical circumstance. An assessment of this type concerns the controllability of quantum systems, and studies exist [7, 8, 9, 10, 11] based on the spectrum of the system field free Hamiltonian along with the coupling interaction to the control field. However, the treatment when the spectrum has degeneracies remains incomplete with the existing approaches either attempting to lift the degeneracy [8] or considering special coupling operators [10]. In particular, neither treatment permits the fundamental analysis of controllability of pure molecular rotational motion. This work addresses this situation in the context of dipole coupling and obtains positive controllability results. In addition, the analysis takes into consideration multipolarization fields. This paper is relevant to other (non)-sudden alignment / orientation works $[12,13,14,15]$ and can also be seen as a step towards extending the treatment to a general assessment of controllability of vibration-rotation states. An additional related topic is rotational control through non-resonant polarization interactions [16], which will be treated in a separate work.

\section{Physical picture}

Consider a linear rigid molecule described by the Hamiltonian $H=B \hat{J}^{2}$ where $B$ is the rotational constant and $\hat{J}$ the is the angular momentum operator. The molecule's rotational motion is subject to control through external interactions with an electric field $\overrightarrow{\epsilon(t)}$, which couples to the molecule through the dipole operator $\vec{d}$. The time dependent Schrödinger equation is

$$
\begin{aligned}
& i \hbar \frac{\partial}{\partial t}|\psi(\theta, \phi, t)\rangle=\left(B \hat{J}^{2}-\overrightarrow{\epsilon(t)} \cdot \vec{d}\right)|\psi(\theta, \phi, t)\rangle \\
& |\psi(0)\rangle=\left|\psi_{0}\right\rangle,
\end{aligned}
$$

where $\theta$ and $\phi$ are the standard polar coordinates. We consider that the external field $\overrightarrow{\epsilon(t)}$ is multi-polarized i.e. any of its $x, y, z$ components can be tuned independently as a function of time. See Section 4 for discussion of other situations.

For convenience we express the problem in spherical harmonics $\left|Y_{J}^{m}\right\rangle, J \geq 0$ and $-J \leq m \leq J$ as the eigenbasis of the operator $H=B \hat{J}^{2}$ such that $B \hat{J}^{2}\left|Y_{J}^{m}\right\rangle=E_{J}\left|Y_{J}^{m}\right\rangle$, $E_{J}=B J(J+1)$. The difference between two consecutive eigenvalues $E_{J+1}-E_{J}=$ $2 B(J+1)$ increases with $J$. Thus, beyond some threshold value of $J$ depending on the control field characteristics, this gap will lie outside of the available frequency range of the field in any practical situation. As such, we will truncate the set of spherical harmonics $\left|Y_{J}^{M}\right\rangle$ at a suitable value $J_{\max }$ and consider control over the domain of states $J \leq J_{\max }$ 
Remark 1 The truncation is supported here by the convenient spectral properties of the operator $B \hat{J}^{2}$ having only discrete eigenvalues which become increasingly sparse as the energy increases. In particular, no accumulation of the discrete spectrum towards a continuous spectral region is present. However, the impact of truncation in other contexts is not always transparent for assessing controllability. For instance, any finite dimensional truncation of the linearly driven harmonic oscillator [10, 17] is controllable, but the infinite dimensional system is not. In that situation the gaps between consecutive eigenvalues are all equal, and a sufficiently long external control pulse can lead to excitation of states indefinitely high in the spectrum.

Remark 2 A circumstance may occur when the wavefunction is stimulated to populate eigenfunctions of increasing energy and even go to dissociation [6]; the field frequency increases indefinitely in order to address this increasing energy gap between two consecutive eigenvalues. This situation is beyond the treatment here that only considers bound states remaining within a given energy range.

The dipole interaction $\overrightarrow{\epsilon(t)} \cdot \vec{d}$ may be expressed as $\epsilon_{x}(t) x+\epsilon_{y}(t) y+\epsilon_{z}(t) z$ in terms of space fixed cartesian coordinates $\vec{x}, \vec{y}$ and $\vec{z}$, where each of the components $\epsilon_{x}(t), \epsilon_{y}(t), \epsilon_{z}(t)$ of the field can be tuned independently.

The $J=1$ spherical harmonics

$$
Y_{1}^{ \pm 1}=\frac{\mp 1}{2} \sqrt{\frac{3}{2 \pi}} \frac{x \pm i y}{r}, Y_{1}^{0}=\frac{1}{2} \sqrt{\frac{3}{\pi}} \frac{z}{r},
$$

may be written in the rotated frame $\frac{\vec{x}+i \vec{y}}{\sqrt{2}}, \frac{\vec{x}-i \vec{y}}{\sqrt{2}}$ and $\vec{z}$. Choosing this latter frame we obtain $\overrightarrow{\epsilon(t)} \cdot \vec{d}=\epsilon_{0}(t) d_{10} Y_{1}^{0}+\epsilon_{+1}(t) d_{11} Y_{1}^{1}+\epsilon_{-1}(t) d_{1-1} Y_{1}^{-1}$. For convenience the components $d_{10}, d_{11}, d_{1-1}$ of $\vec{d}$ are assumed to be all nonzero and will be rescaled to 1 (this is equivalent to rescaling the components $\epsilon_{0}, \epsilon_{+1}$ and $\epsilon_{-1}$ of $\overrightarrow{\epsilon(t)}$ ) so that we obtain $\overrightarrow{\epsilon(t)} \cdot \vec{d}=\epsilon_{0}(t) Y_{1}^{0}+\epsilon_{+1}(t) Y_{1}^{1}+\epsilon_{-1}(t) Y_{1}^{-1}$

Let $D_{k}$ be the matrix of the spherical harmonic operator $Y_{1}^{k}(k=-1,0,1)$. The associated matrix elements may be written as [18]:

$$
\begin{aligned}
& \left(D_{k}\right)_{(J m),\left(J^{\prime} m^{\prime}\right)}=\left\langle Y_{J}^{m}\left|Y_{1}^{k}\right| Y_{J^{\prime}}^{m^{\prime}}\right\rangle=\int\left(Y_{J}^{m}\right)^{*}(\theta, \phi) Y_{1}^{k}(\theta, \phi) Y_{J^{\prime}}^{m^{\prime}}(\theta, \phi) \sin (\theta) d \theta d \phi \\
& =\sqrt{\frac{3(2 J+1)\left(2 J^{\prime}+1\right)}{4 \pi}}\left(\begin{array}{ccc}
J & 1 & J^{\prime} \\
0 & 0 & 0
\end{array}\right)\left(\begin{array}{ccc}
J & 1 & J^{\prime} \\
m & k & m^{\prime}
\end{array}\right) .
\end{aligned}
$$

where the real constants $\left(\begin{array}{ccc}J & 1 & J^{\prime} \\ 0 & 0 & 0\end{array}\right)$ and $\left(\begin{array}{ccc}J & 1 & J^{\prime} \\ m & k & m^{\prime}\end{array}\right)$ are Wigner 3J-symbols (see [19] Chapter 2 for formulas and details). The non-zero elements satisfy the criteria $m+k+m^{\prime}=0$ and $\left|J-J^{\prime}\right|=1$. Thus, the only non-zero entries of the matrix $D_{-1}$ are between states $\left|Y_{J}^{m}\right\rangle$ and $\left|Y_{J+1}^{-m+1}\right\rangle$; we will say that $D_{-1}$ couples states $\left|Y_{J}^{m}\right\rangle$ and $\left|Y_{J+1}^{-m+1}\right\rangle$; similarly $D_{0}$ only couples $\left|Y_{J}^{m}\right\rangle$ and $\left|Y_{J+1}^{-m}\right\rangle$ and $D_{1}$ only couples $\left|Y_{J}^{m}\right\rangle$ and $\left|Y_{J+1}^{-m-1}\right\rangle$. 


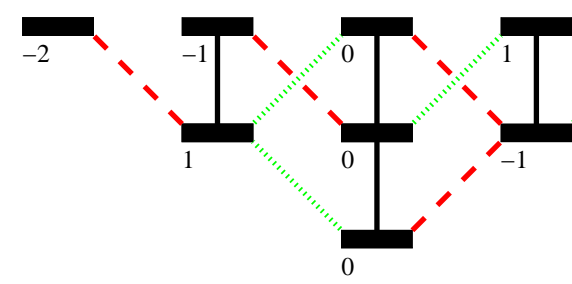

$\mathrm{J}=2$

$\mathrm{J}=1$

$\mathrm{J}=0$

Figure 1. The three matrices $D_{k}, k=-1,0,1$ coupling the eigenstates are each represented by a different line style (dotted, solid and dashed) for $J_{\max }=2$. On the $J$-th line from bottom, the states are from left to right in order $\left|Y_{J}^{m=-J}\right\rangle, \ldots,\left|Y_{J}^{m=J}\right\rangle$ for even values of $J$ and $\left|Y_{J}^{m=J}\right\rangle, \ldots,\left|Y_{J}^{m=-J}\right\rangle$ for odd values of $J$. The $m$ quantum number labelings are indicated in the figure. The coupling pattern above continues in a similar fashion for $J_{\max }>2$.

Denoting by $\Psi(t)$ the coefficients of $\psi(\theta, \phi, t)$ with respect to the spherical harmonic basis, the Schrödinger equation in matrix form is

$$
\left\{\begin{array}{l}
i \frac{\partial}{\partial t} \Psi(t)=\left(E-\epsilon_{0}(t) D_{0}-\epsilon_{-1}(t) D_{-1}-\epsilon_{1}(t) D_{1}\right) \Psi(t) \\
\Psi(t=0)=\Psi_{0} .
\end{array}\right.
$$

where $E$ is the diagonal matrix with entries $E_{J}$ for all dual indexes $J m$ with $-J \leq m \leq J$ and $J \leq J_{\max }$.

\section{Controllability assessment with three independently polarized field components}

We desire to determine whether the system is controllable over all of its $J$ and $m$ states up to $J_{\max }$ provided that all of the control field components $\epsilon_{k}(t) k=-1,0,1$ can be chosen independently. Intuitive arguments were given in [15] (pages 437-438) on why the answer to this question should be positive for all truncation values $J_{\max }$. The material below provides a rigorous grounding for this claim.

Theorem 1 Let $J_{\max } \geq 1$ and denote $N=\left(J_{\max }+1\right)^{2}$. Let $E, D_{k}, k=-1,0,1$ be $N \times N$ matrices indexed by $J m$ with $J=0, \ldots, J_{\text {max }},|m| \leq J$ where:

$$
\begin{aligned}
& E_{J m ; J^{\prime} m^{\prime}}=\delta_{J J^{\prime}} \delta_{m m^{\prime}} E_{J} \\
& \left(D_{0}\right)_{J m, J^{\prime} m^{\prime}} \neq 0 \Leftrightarrow\left|J-J^{\prime}\right|=1, m+m^{\prime}=0 \\
& \left(D_{1}\right)_{J m, J^{\prime} m^{\prime}} \neq 0 \Leftrightarrow\left|J-J^{\prime}\right|=1, m+m^{\prime}+1=0 \\
& \left(D_{-1}\right)_{J m, J^{\prime} m^{\prime}} \neq 0 \Leftrightarrow\left|J-J^{\prime}\right|=1, m+m^{\prime}-1=0 .
\end{aligned}
$$

and recall that

$$
E_{J}=J(J+1) .
$$

Then the system described by $E, D_{-1}, D_{0}, D_{1}$ is controllable.

Proof. In view of the criterion in [7] we have to prove that the Lie algebra $L$ generated by $i E$ and $i D_{k} k=-1,0,1$ is $u(N)$. 
We label by $e_{(a b)}$ the $N \times N$ matrix whose entry at row $a$ and column $b$ is 1 and all others are zero and denote $V_{(a b)}=i\left(e_{(a b)}+e_{(b a)}\right), S_{(a b)}=\left(e_{(a b)}-e_{(b a)}\right)$ and $\Delta_{a}=i\left(e_{(a a)}-e_{(a+1 ; a+1)}\right)$. Note that $S_{(a b)}, V_{(a b)},(a<b, a, b=1, N)$ and $\Delta_{a}$ $(a=1, \ldots, N-1)$ form a basis for $s u(N)$. We index by $\xi \in \Xi$ (of cardinality $K$ ) the entries $\xi=(a b), a<b$ such that at least one matrix $D_{k}$ has a non-zero entry $\left(D_{k}\right)_{\xi=(a b)} \neq 0$ and denote by $\xi^{\dagger}$ the pair $(b a)$.

We will index the matrices $E$ and $D_{k}$ with $a, b$ running from 1 to $N: E=(E)_{a, b=1}^{N}$, $D_{k}=\left(D_{k}\right)_{a, b=1}^{N}, k=-1,0,1$ where we choose the order for $(J m):(00)$ corresponds to $a=1,(11)$ corresponds to $a=2,(10)$ to $a=3,(1-1)$ to $a=4$, then $(2-2),(2-1),(20),(21),(22), \ldots$ etc (see Fig 1$)$. Note that $(J m)$ traverses from $m=-J$ to $m=J$ for even values of $J$ and from $m=J$ down to $m=-J$ for odd values of $J$. For instance $E_{44}=E_{(1-1),(1-1)}=2$. When there is no ambiguity we will use interchangeably $(1-1)$ or 4 , etc.

For $k=-1,0,1$ and $\ell \geq 1$ we compute $a d_{i E}^{\ell} i D_{k}=\left[i E, \ldots,\left[i E, i D_{k}\right] \ldots\right]=$ $\left(i^{\ell+1} \omega_{a b}^{\ell}\left(D_{k}\right)_{a b}\right)_{a, b=1}^{N}\left(\omega_{a b}=E_{a a}-E_{b b}\right)$ with the iterative commutators taken $\ell$ times. Consider the basis

$$
\begin{aligned}
& \left\{v_{k}^{J}=\left(\sum_{\xi=(a b), \omega_{\xi}=E_{J+1}-E_{J} ; a<b ;\left(D_{k}\right)_{\xi} \neq 0}\left(D_{k}\right)_{\xi} e_{\xi}\right),\right. \\
& \left.v_{k}^{J \dagger}=\left(\sum_{\xi=(a b), \omega_{\xi}=E_{J+1}-E_{J} ; a<b ;\left(D_{k}\right)_{\xi} \neq 0}\left(D_{k}\right)_{\xi^{\dagger}}^{*} e_{\xi^{\dagger}}\right) ; J=0, \ldots, J_{\max }\right\}
\end{aligned}
$$

and note that $a d_{i E}^{\ell} i D_{k}=\sum_{k, J} i^{\ell+1} \omega_{\xi}^{\ell} v_{k}^{J}+i^{\ell+1}(-1)^{\ell}\left(\omega_{\xi}\right)^{\ell} v_{k}^{J \dagger}$. We obtain as in [20] that, since $\omega_{\xi}$ are all different, $a d_{i E}^{\ell} i D_{k}$ generates any vector in the linear space

$$
\begin{aligned}
& V e c t\left\{\left(\sum_{\xi=(a b), \omega_{\xi}=E_{J+1}-E_{J} ; a<b ;\left(D_{k}\right)_{\xi} \neq 0}\left(D_{k}\right)_{\xi} S_{\xi}\right),\right. \\
& \left.\left.\sum_{\xi=(a b), \omega_{\xi}=E_{J+1}-E_{J} ; a<b ;\left(D_{k}\right)_{\xi} \neq 0}\left(D_{k}\right)_{\xi^{\dagger}}^{*} V_{\xi^{\dagger}}\right) ; J=0, \ldots, J_{\max }\right\} .
\end{aligned}
$$

In particular,

$$
\left(\sum_{\xi=(a b), \omega_{\xi}=E_{J+1}-E_{J} ; a<b ;\left(D_{k}\right)_{\xi} \neq 0}\left(D_{k}\right)_{\xi} S_{\xi}\right) \text { and }\left(\sum_{\xi=(a b), \omega_{\xi}=E_{J+1}-E_{J} ; a<b ;\left(D_{k}\right)_{\xi} \neq 0}\left(D_{k}\right)_{\xi^{\dagger}}^{*} V_{\xi^{\dagger}}\right)
$$

will belong to $L, J=0, \ldots, J_{\max }$.

For $J=0$ we obtain $S_{(a=(00), b=(10))}, S_{(a=(00), b=(1-1))}, S_{(a=(00), b=(11))} \in L$ and the same for $V_{(a=(00), b=(1 m))}, m=-1,0,1$. We recall now the relations

for $a \neq b \neq c \neq a:\left[S_{(a b)}, S_{(b c)}\right]=S_{(a c)},\left[S_{(a b)}, V_{(b c)}\right]=V_{(a c)}$,

for $a, b, a^{\prime}, b^{\prime}$ all different : $\left[S_{(a b)}, S_{\left(a^{\prime} b^{\prime}\right)}\right]=0,\left[S_{(a b)}, V_{\left(a^{\prime} b^{\prime}\right)}\right]=0$,

for $a=1, \ldots, N-1:\left[S_{(a, a+1)}, V_{(a, a+1)}\right]=\Delta_{a}$, 
and note that the commutators

$$
\left[S_{(a=(00), b)},\left(\sum_{\xi=\left(b^{\prime} c^{\prime}\right), \omega_{\xi}=E_{2}-E_{1} ; b^{\prime}<c^{\prime} ;\left(D_{k}\right)_{\xi} \neq 0}\left(D_{k}\right)_{\xi} S_{\xi}\right)\right]
$$

contain only one term $\left(D_{k}\right)_{(b c)} S_{(a c)}$ where $c=\left(2 c_{2}\right)$ is the (level $J=2$ ) state coupled with state $b=\left(1 b_{2}\right)$ (level $J=1$ ) through the same matrix $D_{k}$ that also couples the state $a$ (level $J=0)$ and $b:\left(D_{k}\right)_{(a b)} \neq 0 \neq\left(D_{k}\right)_{(b c)}$. Thus $S_{(a c)} \in L$ and, by using the commutator of $S_{(a b)}$ and $S_{(a c)}$, we obtain that $S_{(b c)}$ is in $L$ as well for any $\xi=\left(b=(1 m), c=\left(2 m^{\prime}\right)\right)$ such that $\left(D_{k}\right)_{\xi} \neq 0$ for some $k=-1,0,1$. It remains now to iterate the above treatment for all levels $J=2, \ldots, J_{\max }$ to obtain that all $S_{\xi}$ and $V_{\xi}$ coupled by some matrix $k:\left(D_{k}\right)_{\xi} \neq 0$ are in $L$. We note that the graph $[8,9,11]$ of the system is connected and conclude that $L=u(N)$.

Remark 3 The same conclusion as that of Thm. 1 holds if one replaces (10) by the more general condition

$$
E_{J+1}-E_{J} \neq E_{J^{\prime}+1}-E_{J^{\prime}}, \forall J \neq J^{\prime}
$$

Theorem 2 Consider a finite dimensional system where a set of states, indexed as $a=(J m)$ with $J=0, \ldots, J_{\max }, m=1, \ldots, m_{J}^{\max }, m_{0}^{\max }=1$, are such that

$$
\begin{aligned}
& E_{J m ; J^{\prime} m^{\prime}}=\delta_{J J^{\prime}} \delta_{m m^{\prime}} E_{J} \\
& E_{J+1}-E_{J} \neq E_{J^{\prime}+1}-E_{J^{\prime}}, \forall J \neq J^{\prime} .
\end{aligned}
$$

We also introduce the set of $K$ external interactions with corresponding matrices $D_{k}$, $k=1, \ldots, K$ where $D_{k}$ only couples states $(J m)$ and $\left(J^{\prime} m^{\prime}\right)$ such that $\left|J-J^{\prime}\right|=1$ and only one non-zero coupling exists for any $(\mathrm{Jm})$ :

$$
\begin{aligned}
& \left(D_{k}\right)_{(J m),\left(J^{\prime} m^{\prime}\right)} \neq 0 \Rightarrow\left|J-J^{\prime}\right|=1 \\
& \left(D_{k}\right)_{(J m),\left(J^{\prime} m^{\prime}\right)} \neq 0,\left(D_{k}\right)_{(J m),\left(J^{\prime \prime} m^{\prime \prime}\right)} \neq 0, J \leq J^{\prime} \leq J^{\prime \prime} \Rightarrow J^{\prime}=J^{\prime \prime}, m^{\prime}=m^{\prime \prime}
\end{aligned}
$$

We also suppose that the graph [8, 9, 11] of the system is connected. Then the system described by $E_{J}, D_{k}\left(J=0, \ldots, J_{\max }, k=1, \ldots, K\right)$ is controllable.

Proof. Under the assumptions above, the proof follows exactly the same path as the one of the Thm. 1

Remark 4 The transition energy condition in Eqns. (14) and (16) is consistent with the rotation of a linear rigid molecule. In addition the further flexibility encompasses broader circumstances including the possibility of hindered rotation of a molecule residing in a trapped nanoscale environment. Moreover, when hypothesis (15) is not satisfied because the system is not degenerate previous results apply [8, 9, 10, 11]. 
Remark 5 The results above can be extended to the case of a symmetric top molecule; in such a circumstance [18] the energy levels are described by three quantum numbers $E_{J K m}$ with $|m| \leq J,|K| \leq J$ and

$$
E_{J K m}=C_{1} J(J+1)+C_{2} K^{2},
$$

for some constants $C_{1}$ and $C_{2}$. If the initial state is in the ground state, or any other state with $K=0$ the coupling operators have the same structure as in Thm. 1 and thus any linear combination of eigenstates with quantum numbers $J, K=0, m$ can be reached (same result directly applies). A more detailed analysis of symmetric top molecules will be presented in a future work.

\section{Controllability for a locked combination of lasers}

We consider here whether the positive result above is still true when $\epsilon_{k}(t), k=-1,0,1$ are not chosen independently but with a locked linear dependence through coefficients $\alpha_{k}$ such that $\overrightarrow{\epsilon(t)} \cdot \vec{d}=\epsilon(t)\left\{\alpha_{-1} Y_{1}^{-1}+\alpha_{0} Y_{1}^{0}+\alpha_{1} Y_{1}^{1}\right\}$. Note that there may exist cases that are not controllable for any given linear combination. One such example is :

$$
E=\left(\begin{array}{cccc}
0 & 0 & 0 & 0 \\
0 & 2 & 0 & 0 \\
0 & 0 & 2 & 0 \\
0 & 0 & 0 & 2
\end{array}\right), \overrightarrow{e(t)} \cdot \vec{d}=\epsilon(t) \mu, \mu=\left(\begin{array}{cccc}
0 & \alpha_{-1} & \alpha_{0} & \alpha_{1} \\
\alpha_{-1} & 0 & 0 & 0 \\
\alpha_{0} & 0 & 0 & 0 \\
\alpha_{1} & 0 & 0 & 0
\end{array}\right)(20)
$$

This system is such that for all $\alpha_{k}(k=-1,0,1)$ the Lie algebra generated by $i E$ and $i \mu$ is $u(2)$, thus the system is not controllable with one laser field. However, Thm. 1 shows that it will become controllable provided that the three components $\epsilon_{k}(t), k=-1,0,1$ can be chosen independently.

The following result describes this situation further.

Theorem 3 Let $A, B_{1}, \ldots, B_{K}$ be elements of a finite dimensional Lie algebra $L$. For $\alpha=$ $\left(\alpha_{1}, \ldots, \alpha_{K}\right) \in \mathbb{R}^{K}$ we denote $L_{\alpha}$ as the Lie algebra generated by $A$ and $B_{\alpha}=\sum_{k=1}^{K} \alpha_{k} B_{k}$. Define the maximal dimension of $L_{\alpha}$

$$
d_{A, B_{1}, \ldots, B_{K}}^{1}=\max _{\alpha \in \mathbb{R}^{K}} \operatorname{dim}_{\mathbb{R}}\left(L_{\alpha}\right) .
$$

Then with probability one with respect to $\alpha, \operatorname{dim}\left(L_{\alpha}\right)=d_{A, B_{1}, \ldots, B_{K}}^{1}$.

Remark 6 This theorem states that for fixed $A, B_{1}, \ldots, B_{K}$ all choices of $\alpha$ give a Lie algebra $L_{\alpha}$ of maximal dimension with the possible exception of at most a null measure set. This dimension $d_{A, B_{1}, \ldots, B_{K}}^{1}$ is specific to the choice of coupling operators $B_{k}$ but can be easily computed by the property above. On the other hand, recall that [21] when $A, B_{1}, \ldots, B_{K}$ are $r \times r$ skew-hermitian matrices the system is generically controllable i.e., we have $d_{A, B_{1}, \ldots, B_{K}}^{1}=r^{2}$ for generic $A, B_{1}, \ldots, B_{K}$.

Proof. Consider the (countable) collection $\mathcal{C}^{\alpha}=\left\{\zeta_{1}^{\alpha}=A, \zeta_{2}^{\alpha}=B, \zeta_{3}^{\alpha}=\left[A, B_{\alpha}\right], \zeta_{4}^{\alpha}=\right.$ $\left.\left[B_{\alpha}, A\right], \zeta_{5}^{\alpha}=\left[A,\left[A, B_{\alpha}\right]\right], \ldots\right\}$ listing all possible iterative commutators constructed from 
$A$ and $B_{\alpha}$. Now take a subset $\left\{\zeta_{i_{1}}^{\alpha}, \ldots, \zeta_{i_{r}}^{\alpha}\right\}$ of $\mathcal{C}^{\alpha}$; the vectors $\zeta_{i_{1}}^{\alpha}, \ldots, \zeta_{i_{r}}^{\alpha}$ are linearly independent when the Gram determinant is non-null. Note that the Gram determinant is an analytic function of $\alpha$; hence one of the following alternatives is true: either this function is identically null for all $\alpha$ (which is the case e.g., for $\left\{\zeta_{3}^{\alpha}, \zeta_{4}^{\alpha}\right\}$ ) or it is nonnull everywhere with the possible exception of a zero measure set. Since the number of subsets $\left\{\zeta_{i_{1}}^{\alpha}, \ldots, \zeta_{i_{r}}^{\alpha}\right\}$ of $\mathcal{C}^{\alpha}$ is countable, we can construct $\mathcal{F} \subset \mathbb{R}^{K}$ whose complement $\mathbb{R}^{K} \backslash \mathcal{F}$ is of zero measure such that if $\zeta_{i_{1}}^{\alpha}, \ldots, \zeta_{i_{r}}^{\alpha}$ are linearly independent for one value of $\alpha \in \mathbb{R}^{K}$ then they are linearly independent for all $\alpha^{\prime} \in \mathcal{F}$. Denote by $\alpha^{\star}$ some value such that $\operatorname{dim}_{\mathbb{R}}\left(L_{\alpha^{\star}}\right)=d_{A, B_{1}, \ldots, B_{K}}^{1}$; then there exists a set such that $\left\{\zeta_{i_{1}}^{\alpha^{\star}}, \ldots, \zeta_{i_{A, B_{1}, \ldots, B_{K}}^{\alpha^{\star}}}^{\alpha^{1}}\right\}$ are linearly independent implying that $\left\{\zeta_{i_{1}}^{\alpha^{\star}}, \ldots, \zeta_{i_{M}^{1}}^{\alpha^{\star}}\right\}$ are linearly independent for any $\alpha \in \mathcal{F}$; thus $\operatorname{dim}_{\mathbb{R}}\left(L_{\alpha}\right) \geq d_{A, B_{1}, \ldots, B_{K}}^{1}$ for all $\alpha \in \mathcal{F}$ and the conclusion follows by the maximality of $d_{A, B_{1}, \ldots, B_{K}}^{1}$.

We invoked Remark 6 and performed numerical tests by computing the Lie algebra generated by $i E$ and $i D_{\alpha}=i \sum_{k=-1}^{1} \alpha_{k} D_{k}$. The theorem was verified and we obtained for any fixed $J_{\max }$ and randomly chosen values of $\alpha$ that the dimensions of the Lie algebra are the same. We also observed that the Lie algebra generated by $i E$ and $i \sum_{k=-1}^{1} \alpha_{k} D_{k}$ always had dimension $(N-2)^{2}$ which leads to the conjecture that generically in $\alpha$ (see Thm 3) the Lie algebra generated by $i E$ and $i \sum_{k=-1}^{1} \alpha_{k} D_{k}$ is isomorphic to $u(N-2)$. Recall that by Thm. 1 when three independent control intensities are allowed then this Lie algebra is $u(N)$. We have as yet no theoretical explanation of why this appears to be true. This observation shows, nevertheless, the extent to which a locked set of laser intensities is sufficient to obtain specific attainable control targets.

\section{Controllability with two lasers}

We consider in this section the situation when two laser fields are used i.e. one can independently shape the intensity along two vectors $\vec{\alpha}$ and $\vec{\beta}: \overrightarrow{\epsilon(t)} \cdot \vec{d}=$ $\epsilon_{\alpha}(t)\left\{\alpha_{-1} Y_{1}^{-1}+\alpha_{0} Y_{1}^{0}+\alpha_{1} Y_{1}^{1}\right\}+\epsilon_{\beta}(t)\left\{\beta_{-1} Y_{1}^{-1}+\beta_{0} Y_{1}^{0}+\beta_{1} Y_{1}^{1}\right\}$. Examples of such situations are shaping along $\vec{x}$ and $\vec{z}$ directions, $\vec{x}$ and $\vec{y}$ directions or any other two independent vectors.

Theorem 4 Let $A, B_{1}, \ldots, B_{K}$ be elements of a finite dimensional Lie algebra $L$. We denote for $\alpha=\left(\alpha_{1}, \ldots, \alpha_{K}\right) \in \mathbb{R}^{K}$ and $\beta=\left(\beta_{1}, \ldots, \beta_{K}\right) \in \mathbb{R}^{K}$ by $L_{\alpha, \beta}$ the Lie algebra generated by $A, B_{\alpha}=\sum_{k=1}^{K} \alpha_{k} B_{k}$ and $B_{\beta}=\sum_{k=1}^{K} \beta_{k} B_{k}$.

Define the maximal dimension of $L_{\alpha}$

$$
d_{A, B_{1}, \ldots, B_{K}}^{2}=\max _{\alpha \in \mathbb{R}^{K}} \operatorname{dim}_{\mathbb{R}}\left(L_{\alpha, \beta}\right) .
$$

Then with probability one with respect to $\alpha, \beta, \operatorname{dim}\left(L_{\alpha, \beta}\right)=d_{A, B_{1}, \ldots, B_{K}}^{2}$.

Proof. The proof is similar to that of Thm.3.

This theorem states that all choices of $\alpha, \beta$ give the maximal Lie algebra dimension

$\operatorname{dim}\left(L_{\alpha, \beta}\right)=d_{A, B_{1}, \ldots, B_{K}}^{2}$ with the possible exception of at most a null measure set. We will analyze in the following two particular cases. 


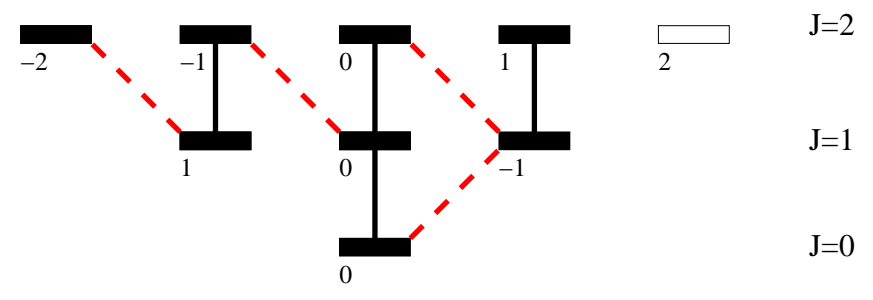

Figure 2. The same conventions as in Fig. 1 are used except that we do not draw on the coupling by $D_{-1}$. We note that the state $\left|Y_{J_{\max }}^{m=J_{\max }}\right\rangle$ is not connected with the others.

5.1. Field shaped in the $\vec{z}$ and $\frac{\vec{x}+i \vec{y}}{\sqrt{2}}$ directions

When the field is shaped in the $\vec{z}$ and $\frac{\vec{x}+i \vec{y}}{\sqrt{2}}$ directions we obtain, with the notation of previous sections, that $\epsilon_{0}(t)$ and $\epsilon_{1}(t)$ are arbitrary and $\epsilon_{-1}$ is null. This is not a generic case in the sense of Thm. 4 because the shaping directions are precisely related to the system structure. We note that since $\epsilon_{-1}=0$ the coupling realized by the operator $D_{-1}$ (dotted in Fig. 1) disappears and the state $\left|Y_{J_{\max }}^{\max }\right\rangle$ will not be reachable (see Fig. 2). This means that the population in state $\left|Y_{J_{\max }}^{\max }\right\rangle$ cannot be changed by the two lasers and thus will be a conserved quantity.

Theorem 5 Consider the model of Thm.1 with $\epsilon_{-1}=0$. Let $\left|\psi_{I}\right\rangle$ and $\left|\psi_{F}\right\rangle$ be two states that have the same population in $\left|Y_{J_{\max }}^{\max }\right\rangle$ i.e., $\left|\left\langle\psi_{I}, Y_{J_{\max }}^{\max }\right\rangle\right|^{2}=\left|\left\langle\psi_{F}, Y_{J_{\max }}^{\max } J_{\max }\right\rangle\right|^{2}$. Then $\left|\psi_{F}\right\rangle$ can be reached from $\left|\psi_{I}\right\rangle$ with controls $\epsilon_{0}(t)$ and $\epsilon_{1}(t)$.

Proof: The conclusion is a consequence of Thm. 2 for $K=2$ and using all spherical harmonics $\left|Y_{J}^{m}\right\rangle$ except $\left|Y_{J_{\max }}^{\max }\right\rangle$. Thus we conclude that the Lie algebra has dimension $N^{2}$ and by the independent system controllability criterion in $[22,23]$ we obtain the conclusion.

A similar analysis applies when the field is shaped in the $\vec{z}$ and $\frac{\vec{x}-i \vec{y}}{\sqrt{2}}$ directions with the modification that in this case the population of $\left|Y_{J_{\max }}^{\max }\right\rangle$ is conserved and the compatibility relation reads:

$$
\left|\left\langle\psi_{I}, Y_{J_{\max }}^{\max =-J_{\max }}\right\rangle\right|^{2}=\left|\left\langle\psi_{F}, Y_{J_{\max }}^{\max } J_{\max }\right\rangle\right|^{2} .
$$

\subsection{Field shaped in the $\frac{\vec{x}+i \vec{y}}{\sqrt{2}}$ and $\frac{\vec{x}-i \vec{y}}{\sqrt{2}}$ directions}

Using the same notation, now $\epsilon_{-1}(t)$ and $\epsilon_{1}(t)$ are arbitrary and $\epsilon_{0}$ is null. Thus, the states are divided in two components cf. Fig. 3: $X_{1}=\left\{\left|Y_{0}^{0}\right\rangle,\left|Y_{1}^{ \pm 1}\right\rangle,\left|Y_{2}^{ \pm 2}\right\rangle,\left|Y_{2}^{0}\right\rangle, \ldots\right\}$ and $X_{2}=\left\{\left|Y_{1}^{0}\right\rangle,\left|Y_{2}^{ \pm 1}\right\rangle,\left|Y_{3}^{ \pm 2}\right\rangle,\left|Y_{3}^{0}\right\rangle, \ldots\right\}$ with no coupling between the two components.

The conservation law reads

$$
\sum_{\left|Y_{J}^{m}\right\rangle \in X_{1}}\left|\left\langle\psi_{I}, Y_{J}^{m}\right\rangle\right|^{2}=\sum_{\left|Y_{J}^{m}\right\rangle \in X_{1}}\left|\left\langle\psi_{F}, Y_{J}^{m}\right\rangle\right|^{2} .
$$




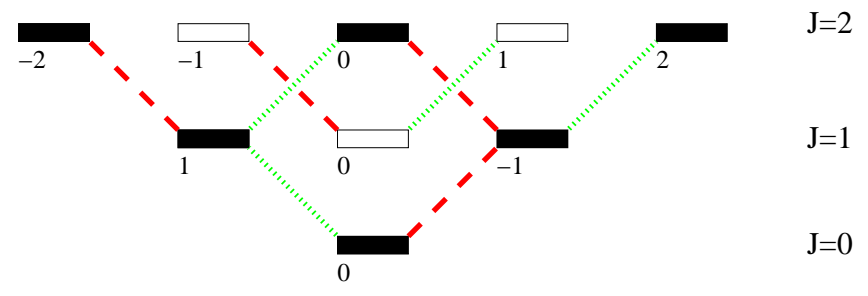

Figure 3. The same conventions as in Fig. 1 are used except that we do not draw on the coupling by $D_{0}$. We note that two connectivity sets appear: those connected with $\left|Y_{0}^{0}\right\rangle$ (filled black rectangles) and those connected with $\left|Y_{1}^{0}\right\rangle$ (empty rectangles).

Theorem 6 Consider the model of the Thm.1 with $\epsilon_{-1}=0$. Let $\left|\psi_{I}\right\rangle$ and $\left|\psi_{F}\right\rangle$ be two states compatible in the sense of Eqn. (24). Then $\left|\psi_{F}\right\rangle$ can be reached from $\left|\psi_{I}\right\rangle$ with controls $\epsilon_{-1}(t)$ and $\epsilon_{1}(t)$.

Proof: The proof follows that of Thm. 2 except that the system has now two independent graphs instead of one, both satisfying the hypothesis of the theorem. The same computation alows one to generate the full Lie algebra for $X_{1}$ (using that for the graph containing the states $X_{1}$ we have $m_{0}^{\max }=1$ ). Next, one works with the second graph and constructs its associated algebra. The conclusion is obtained by the independent system controllability criterion in $[22,23]$.

\section{Conclusions}

This paper discussed the controllability properties of molecular rotation with multipolarization fields that act through a permanent dipole moment. A first conclusion is that the degeneracy of the energy levels brings no additional restriction on the controllability. Positive results are found for the controllability of an arbitrary number of rotation eigenstates. We also discussed the situation of a symmetric top molecule when the magnetic quantum number $m$ is zero.

The dependence of the controllability result on the the coupling operators is not surprising; however as in [9] the numeric values of the entries of the coupling matrices are not important as soon as they are non-null. Thus, we can say that the controllability depends only on the "selection rules" i.e. on the fact that two states may, or may not, have a non-vanishing coupling through one of the external (laser) interactions.

The situation with one and two polarized fields was also examined based on the generic dimension of the Lie algebra. For the particular situations where the field is

shaped in any combination of two of the three directions $\vec{z}, \frac{\vec{x}+i \vec{y}}{\sqrt{2}}$ and $\frac{\vec{x}-i \vec{y}}{\sqrt{2}}$ we showed that the system is still controllable provided that the target is consistent with the selection rules. Breaking those symmetries would require a third independently shaped pulse.

The specific situation with two fields depends on which dual polarization components are available: if one can shape the polarization in the $\vec{z}$ and $\frac{\vec{x}+i \vec{y}}{\sqrt{2}}$ 
directions everything can be controlled except the population of the state $\mid Y_{J_{\max }=J_{\max }}^{\text {max }}$ (respectively $\left|Y_{J_{\max }=-J_{\max }}^{\max }\right\rangle$ for the $\vec{z}$ and $\frac{\vec{x}-i \vec{y}}{\sqrt{2}}$ directions). If on the contrary the field can be shaped in the $\frac{\vec{x}+i \vec{y}}{\sqrt{2}}$ and $\frac{\vec{x}-i \vec{y}}{\sqrt{2}}$ directions, then the initial and target state have to be compatible in the sense of the selection rules. We note that in both cases with two polarization field components (in the list above) one only has a single compatibility constraint to satisfy. This is to be contrasted with the situation when only one polarization component is available : in this case there are many constraints on the target state (e.g., when only $\epsilon_{0}$ is available the selection rules impose $2 J_{\text {max }}+1$ constraints because $m$ is conserved). In summary, the most substantial increase in controllability (based on our analysis of these particular cases and on the general controllability result) is witnessed when replacing a linear polarized field by a field independently shaped in two directions.

Finally, it is important to place this work in the larger context of molecular, and more generally quantum system, controllability. A pertinent issue is whether any randomly chosen Hamiltonian (i.e., a particular physical system drawn from the stockroom) is likely to be controllable. Building on theoretical results [21], recent numerical work [24] argued that virtually any Hamiltonian-coupling operator (i.e., the dipole) expressed in the eigenbasis of the field free Hamiltonian $H_{0}$ will generate a connected graph (e.g., as in Fig. 1 versus that in Fig. 2). Although this statement is short of establishing controllability, it is a necessary criteria. Furthermore, to arrange a special relationship amongst the Hamiltonian's matrix elements in order to violate controllability is a demand whose solution lies in the null space of all Hamiltonians $[8,11]$. In particular we proved in this paper that this null space does not contain the degenerate Hamiltonian for rotational motion. Thus, although uncontrollable Hamiltonians can be designed, the chance of finding one in the laboratory is very small. This conclusion provides the basis to expect that suitable control fields will virtually always exist yielding high quality results.

\section{Acknowledgments}

G.T. acknowledges support from the French ANR C-QUID project, INRIA Rocquencourt (MicMac and OMQS projects) and PICS-NSF program "Manipulation and identification of quantum phenomena". H.R. acknowledges support from the DoE.

\section{References}

[1] Richard S. Judson and H. Rabitz. Teaching lasers to control molecules. Phys. Rev. Lett, 68:1500, 1992.

[2] A. Assion, T. Baumert, M. Bergt, T. Brixner, B. Kiefer, V. Seyfried, M. Strehle, and G. Gerber. Control of chemical reactions by feedback-optimized phase-shaped femtosecond laser pulses. Science, 282:919-922, 1998.

[3] T.C. Weinacht, J. Ahn, and P.H. Bucksbaum. Controlling the shape of a quantum wavefunction. Nature, 397:233-235, 1999. 
[4] R. Bartels, S. Backus, E. Zeek, L. Misoguti, G. Vdovin, I.P. Christov, M.M. Murnane, and H.C. Kapteyn. Shaped-pulse optimization of coherent emission of high-harmonic soft x-rays. Nature, 406:164-166, 2000.

[5] R. J. Levis, G.M. Menkir, and H. Rabitz. Selective bond dissociation and rearrangement with optimally tailored, strong-field laser pulses. Science, 292:709-713, 2001.

[6] D. M. Villeneuve, S. A. Aseyev, P. Dietrich, M. Spanner, M. Yu. Ivanov, and P. B. Corkum. Forced molecular rotation in an optical centrifuge. Phys. Rev. Lett., 85(3):542-545, Jul 2000.

[7] V. Ramakrishna, M.V. Salapaka, M. Dahleh, H. Rabitz, and A. Peirce. Controllability of molecular systems. Phys. Rev. A, 51 (2):960-966, 1995.

[8] Gabriel Turinici and Herschel Rabitz. Quantum wave function controllability. Chem. Phys., $267: 1-9,2001$.

[9] Gabriel Turinici and Herschel Rabitz. Wavefunction controllability in quantum systems. J. Phys.A., 36:2565-2576, 2003.

[10] S. G. Schirmer, H. Fu, and A.I. Solomon. Complete controllability of quantum systems. Phys. Rev. A, 63:063410, 2001.

[11] C. Altafini. Controllability of quantum mechanical systems by root space decomposition of su(N). J.Math.Phys., 43(5):2051-2062, 2002.

[12] Henrik Stapelfeldt and Tamar Seideman. Colloquium: Aligning molecules with strong laser pulses. Reviews of Modern Physics, 75(2):543, 2003.

[13] D. Sugny, A. Keller, O. Atabek, D. Daems, C. M. Dion, S. Guérin, and H. R. Jauslin. Reaching optimally oriented molecular states by laser kicks. Phys. Rev. A, 69(3):033402, Mar 2004.

[14] A. Keller, C. M. Dion, and O. Atabek. Laser-induced molecular rotational dynamics: A highfrequency Floquet approach. Phys. Rev. A, 61(2):023409, Jan 2000.

[15] R.S. Judson, K.K. Lehmann, H. Rabitz, and W. Warren. Optimal design of external fields for controlling molecular motion: application to rotation. J. Molec. Structure, 223:425-456, June 1990.

[16] R. S. Minns, R. Patel, J. R. R. Verlet, and H. H. Fielding. Optical control of the rotational angular momentum of a molecular Rydberg wave packet. Phys. Rev. Lett., 91(24):243601, Dec 2003.

[17] Mazyar Mirrahimi and Pierre Rouchon. Controllability of quantum harmonic oscillators. IEEE Trans. Automat. Control, 49(5):745-747, 2004.

[18] D.M. Brink and G.R. Satchler. Angular Momentum. Oxford University Press, 1994.

[19] Richard Z. Zare. Angular Momentum. John Wiley \& Sons, 1988.

[20] Gabriel Turinici and Herschel Rabitz. Optimally controlling the internal dynamics of a randomly orientated ensemble of molecules. Phys. Rev. A, 70:063412, 2004.

[21] Nik Weaver. On the universality of almost every quantum logic gate. Journal of Mathematical Physics, 41(1):240-243, 2000.

[22] B. Li, G. Turinici, V. Ramakhrishna, and H. Rabitz. Optimal dynamic discrimination of similar molecules through quantum learning control. Journal of Physical Chemistry B, 106(33):8125$8131,2002$.

[23] G. Turinici, V. Ramakhrishna, B. Li, and H. Rabitz. Optimal discrimination of multiple quantum systems: Controllability analysis. Journal of Physics A: Mathematical and General, 37:273-282, 2003.

[24] Rong Wu, Herschel Rabitz, Gabriel Turinici, and Ignacio Sola. Connectivity analysis of controlled quantum systems. Phys. Rev. A, 70(5):052507, Nov 2004. 